\title{
TELEGRAPH EQUATION IN RANDOM WALK PROBLEM*
}

\author{
V. V. Uchaikin, V. V. Saenko \\ Ulyanovsk State University, Institute for Theoretical Physics, \\ Ulyanovsk, 432700, Russia
}

(Received November 5, 1999; received in final form June 2, 2000)

\begin{abstract}
The isotropic random walk of a particle with a constant speed is considered in the $d$-dimensional space. This process is described by the kinetic equation which has explicit solutions in terms of quadratures or special functions only in the cases $d=1$ and 2. For $d>2$, the two reduced forms of the equation are used: the telegraph equation and the diffusion equation. The latter is usually considered as a rougher approximation than the telegraph one. The numerical investigations performed in this article show that actually the situation is diametrically opposed: for $d \geq 2$ the simple diffusion result turns out to be closer to the exact one than the more complex solution of the telegraph equation. The results are applicable to surface transport and volume transport problem and can be useful for describing the chaotic dynamics of a system in terms of random walks in the phase space.
\end{abstract}

Key words: telegraph equation, moment method, random walk.

PACS number(s): 02.50.-r, 02.50.Ga, 02.60.-x, 05.60.+w

\section{INTRODUCTION}

As is noted in [1], the telegraph equation with the dimensionless time $t$ having the form

$$
\frac{\partial^{2} f}{\partial t^{2}}+\frac{\partial f}{\partial t}=\nu^{2} \frac{\partial^{2} f}{\partial x^{2}}
$$

was written down by Lord Kelvin in connection with the first transatlantic submarine telephone cable [2]. Then it was revealed that Eq. (1) exactly describes a onedimensional walk of a particle with a constant speed and with the random free path distributed according to the exponential law $[3,4]$. Moreover, the telegraph equation describes the time-dependent distribution of one of the coordinates of a particle walking isotropically in a three-dimensional space $[5-7]$ but this time it gives an approximate solution of the problem known as $P_{1}$-approximation [8]. In this case, Eq. (1) is often regarded as a better approximation than the diffusion equation $[9,10]$

$$
\frac{\partial f}{\partial t}=\nu^{2} \frac{\partial^{2} f}{\partial x^{2}}
$$

It is really so in the one-dimensional case when Eq. (1) is fully equivalent to the Boltzman equation exactly describing the distribution of a walking particle.

If the particle starts its walk from the origin $x=0$ at the moment $t=0$, then the distribution $f(x, t)$ given by the solution of Eq. (1) is different from zero in the domain $-\nu t<x<\nu t$ broadening linearly with time. The $x= \pm \nu t$ are points of the diffusion "wave" front beyond $f(x, t)=0$ because the particle with the speed of a free motion being equal to $\nu$ can not reach the point $x$ until $t \geq|x| / \nu$.

However, when the dimension $d>1$ the situation changes. Now $\nu$ means root-mean-square projection $\nu=\sqrt{V_{x}^{2}}$ of the velocity $\mathbf{V}$ on the $x$-axis [5] and Eq. (1) gives the solution equal to zero beyond the interval $(-\nu t, \nu t)$, while in reality the particle may be revealed in the wider domain $(-v t, v t), v=|\mathbf{V}|$. The two results lead to the natural question: how accurate is the telegraph approximation (1) inside the interval as compared with the diffusion approximation (2)? The aim of this article is to answer the question.

\section{THE PROBLEM STATEMENT}

Let us define the random walk problem with exponential pausing time more exactly. Some particle walking in the $d$-dimensional space with the constant speed $v=1$ starts its motion from the origin of coordinates at the time $t=0$ in random direction distributed isotropically over the whole solid angle so that the $x$-projection of the velocity has the probability density $W_{d}\left(v_{x}\right)$ (see App. A). The random time $T$ up to the next scattering is distributed according to the density

$$
p_{T}(t)=e^{-t}, \quad 0 \leq t<\infty
$$

As a result of the collision, the particle changes its motion direction and the new direction is isotropically distributed again independently of the previous one; then the process is repeated. 
Let $X(t)$ be a random value of one of the coordinates of the walking particle at the time $t$ under condition

$$
X(0)=0 .
$$

According to the law of total probability

$$
\begin{aligned}
& \operatorname{Prob}\{x \leq X(t)<x+d x\} \\
& =\operatorname{Prob}\{x \leq X(t)<x+d x \mid T \geq t\} \operatorname{Prob}\{T \geq t\} \\
& +\operatorname{Prob}\{x \leq X(t)<x+d x, T<t\} .
\end{aligned}
$$

The first conditional probability describes the unscattered particle $\left(X(t)=X^{(0)}(t)=V_{x} t\right)$. It is connected to the probability density $W_{d}\left(v_{x}\right)$ via relation

$$
\begin{aligned}
& \operatorname{Prob}\{x \leq X(t)<x+d x \mid T \geq t\} \\
& =\operatorname{Prob}\left\{x / t \leq X^{(0)}(t) / t<x / t+d x / t\right\}=W_{d}(x / t) d x / t .
\end{aligned}
$$

Taking into account that

$$
\operatorname{Prob}\{T \geq t\}=\int_{t}^{\infty} e^{-\tau} d \tau=e^{-t}
$$

and passing to distribution densities

$$
\begin{aligned}
p_{d}(x, t) & =\operatorname{Prob}\{x \leq X(t)<x+d x\} / d x, \\
p_{d}^{(0)}(x, t) & =\operatorname{Prob}\left\{x \leq X^{(0)}(t)<x+d x\right\} / d x \\
& =e^{-t} W_{d}(x / t) / t
\end{aligned}
$$

we rewrite Eq. (3) in the form

$$
p_{d}(x, t)=p_{d}^{(0)}(x, t)+S_{d}(x, t) .
$$

Here, $S_{d}(x, t)$ is the collision integral the explicit expression for which can be obtained regarding $X(t)$ as the sum $X^{0}(T)+X(t-T)$ of two independent (by fixed $T<t$ ) random variables with the densities $W_{d}(x / T) / T$ and $p_{d}(x, t-T)$ correspondingly. Convoluting the densities and averaging over $T$ leads to the following expression:

$S_{d}(x, t)=\int_{0}^{t} d \tau \int_{-\tau}^{\tau} d \xi e^{-\tau}\left[W_{d}(\xi / \tau) / \tau\right] p_{d}(x-\xi, t-\tau)$.

Collecting (4)-(6) we arrive at the integral kinetic equation being the basis for further analysis:

$$
p_{d}(x, t)=p_{d}^{(0)}(x, t)
$$

$$
+\int_{0}^{t} d \tau \int_{-\tau}^{\tau} d \xi p_{d}^{(0)}(\xi, \tau) p_{d}(x-\xi, t-\tau)
$$

\section{REDUCED EQUATIONS}

It is convenient for our purposes to rewrite equation (7) in the Fourier-Laplace space:

$$
\tilde{p}_{d}(k, \lambda)=\tilde{p}_{d}^{(0)}(k, \lambda)\left[1+\tilde{p}_{d}(k, \lambda)\right],
$$

where

$$
\tilde{p}_{d}(k, \lambda)=\int_{0}^{\infty} d t e^{-\lambda t} \int_{-t}^{t} d x e^{i k x} p_{d}(x, t) .
$$

Equation (8) readily yields

$$
\tilde{p}_{d}(k, \lambda)=\frac{\tilde{p}_{d}^{(0)}(k, \lambda)}{1-\tilde{p}_{d}^{(0)}(k, \lambda)}
$$

The Fourier-Laplace transform of the unscattered particle distribution is reduced to the form

$$
\tilde{p}_{d}^{(0)}(k, \lambda)=\frac{1}{k} \int_{0}^{\infty} e^{-(\lambda+1) q / k} \tilde{W}_{d}(q) d q
$$

with

$$
\begin{aligned}
\tilde{W}_{d}(q) & =2 \int_{0}^{1} W_{d}\left(v_{x}\right) \cos \left(q v_{x}\right) d v_{x} \\
& =2^{d / 2-1} \Gamma(d / 2) J_{d / 2-1}(q) q^{1-d / 2}
\end{aligned}
$$

(see (A.9)). Substituting (10) into (9) gives

$$
\tilde{p}_{d}^{(0)}(k, \lambda)=F\left(1 / 2,1 ; d / 2 ;-k^{2} /(\lambda+1)^{2}\right) /(\lambda+1),
$$

where

$$
F(1 / 2,1 ; d / 2 ;-z)=\frac{1}{\sqrt{\pi}} \Gamma\left(\frac{d}{2}\right) \sum_{m=0}^{\infty} \frac{\Gamma(1 / 2+m)}{\Gamma(d / 2+m)}(-z)^{m}
$$

is a hypergeometrical function [11]. In particular

$$
\tilde{p}_{1}^{(0)}(k, \lambda)=\frac{\lambda+1}{(\lambda+1)^{2}+k^{2}},
$$




$$
\begin{aligned}
& \tilde{p}_{2}^{(0)}(k, \lambda)=\frac{1}{\sqrt{(\lambda+1)^{2}+k^{2}}}, \\
& \tilde{p}_{3}^{(0)}(k, \lambda)=\frac{1}{k} \operatorname{arctg} \frac{k}{\lambda+1}, \\
& \tilde{p}_{4}^{(0)}(k, \lambda)=\frac{2}{(\lambda+1)+\sqrt{(\lambda+1)^{2}+k^{2}}} .
\end{aligned}
$$

\section{FROM THE TELEGRAPH TO THE DIFFUSION EQUATION}

It is readily seen that in the one-dimensional case the substitution of (12) in (8) leads to the equation

$$
\left(\lambda^{2}+\lambda+k^{2}\right) \tilde{p}_{1}(k, \lambda)=\lambda+1
$$

Let $g^{(\nu)}(x, t)$ be the solution of the telegraph equation under initial conditions

$$
g^{(\nu)}(x, 0)=\delta(x)
$$

and

$$
\left[\partial g^{(\nu)}(x, t) / \partial t\right]_{t=0}=0
$$

Explicit expressions for $g^{(\nu)}(x, t)$ and its moments are given in Appendix B. The Fourier-Laplace transform of $g^{(\nu)}(x, t)$ obeys the equation

$$
\left(\lambda^{2}+\lambda+\nu^{2} k^{2}\right) \tilde{g}^{(\nu)}(k, \lambda)=\lambda+1
$$

so the comparison of (13) with (14) yields

$$
p_{1}(x, t)=g^{(1)}(x, t)
$$

Thus, in the one-dimensional case the kinetic equation (7) has exactly the same solution as the telegraph equation (1), they both are strictly equivalent.

In a space with $d>1$, the strict equivalence does not take place any more. Using the expansion of the function $1 / F(1 / 2,1 ; d / 2,-z)$ in terms of $z$ :

$$
F(1 / 2,1 ; d / 2 ;-z) \sim \frac{1}{1-z / d}, \quad z \rightarrow 0
$$

and setting this into (11) yields the asymptotic relation

$$
\tilde{p}_{d}^{(0)}(k, \lambda) \sim \frac{\lambda+1}{(\lambda+1)^{2}+k^{2} / d}, \quad k \rightarrow 0
$$

and then

$$
\left(\lambda^{2}+\lambda+k^{2} / d\right) \tilde{p}_{d}^{T}(k, \lambda)=\lambda+1
$$

This is the Fourier-Laplace transform of the equation

$$
\begin{aligned}
& \frac{\partial^{2} p_{d}^{T}(x, t)}{\partial t^{2}}+\frac{\partial p_{d}^{T}(x, t)}{\partial t} \\
& -\frac{1}{d} \frac{\partial^{2} p_{d}^{T}(x, t)}{\partial x^{2}}=0, \quad t>0
\end{aligned}
$$

with the condition

$$
p_{d}^{T}(x, 0)=\delta(x), \quad\left[\partial p_{d}^{T}(x, t) / \partial t\right]_{t=0}=0
$$

where $p_{d}^{T}(x, t)$ denotes solution of the telegraph equation. So

$$
p_{d}^{T}(x, t)=g^{(\nu)}(x, t)
$$

where $g^{(\nu)}(x, t)$ is given by (B1) and

$$
\nu=1 / \sqrt{d}
$$

According to the Tauberian theorem [12] only the region of small $\lambda$ plays an essential role in forming the solution of (15) at a large time. As a result we have

$$
\lambda \tilde{p}_{d}^{D}(k, \lambda)=-\left(k^{2} / d\right) \tilde{p}_{d}^{D}(k, \lambda)+1
$$

which is nothing but the Fourier-Laplace transform of the ordinary diffusion equation

$$
\frac{\partial p_{d}^{D}(x, t)}{\partial t}=\frac{1}{d} \frac{\partial^{2} p_{d}^{D}(x, t)}{\partial x^{2}}, \quad p_{d}^{D}(x, 0)=\delta(x)
$$

with the solution

$$
p_{d}^{D}(x, t)=\frac{1}{\sqrt{4 \pi t / d}} \exp \left\{-x^{2} /(4 t / d)\right\}
$$

\section{ANALYTICAL SOLUTIONS}

Three types of equations describing the process of the random walker with exponential pausing time are introduced above: approximate the diffusion equation (18) with the solution (19), the telegraph equation (15) that is exact for $d=1$, approximate for $d \geq 2$ and that has solution (16), and the kinetic equation (7) exactly describing the process for all $d$. We consider here its solution.

As we saw above, in the one-dimensional case

$$
p_{1}(x, t)=g^{(1)}(x, t) .
$$




\section{V. UCHAIKIN, V. V. SAENKO}

In the two-dimensional case the distribution density $p_{2}(x, t)$ can be expressed through the two-variate density $p_{2}(x, y, t)$ as follows

$$
p_{2}(x, t)=\int_{-\infty}^{\infty} p_{2}(x, y, t) d y
$$

The density $p_{2}(x, y, t)$ is found in [13] and has the form

$$
\begin{aligned}
p_{2}(x, y, t) & =e^{-t}\left[\frac{\delta(r-t)}{2 \pi r}\right. \\
& \left.+\frac{1}{2 \pi \sqrt{t^{2}-r^{2}}} \exp \left(\sqrt{t^{2}-r^{2}}\right) H(t-r)\right]
\end{aligned}
$$

where $r=\sqrt{x^{2}+y^{2}}$ and

$$
H(t)= \begin{cases}0, & t<0 \\ 1, & t \geq 0\end{cases}
$$

is the Heaviside function. Substitution of (21) into (20) yields

$$
\begin{aligned}
p_{2}(x, t) & =\frac{1}{\pi} e^{-t} \\
& \times\left[\frac{1}{\sqrt{t^{2}-x^{2}}}+\int_{0}^{\sqrt{t^{2}-x^{2}}} \frac{e^{\sqrt{t^{2}-x^{2}-\xi^{2}}} d \xi}{\sqrt{\left.t^{2}-x^{2}\right)-\xi^{2}}}\right] .
\end{aligned}
$$

form including a double integral of an oscillating complex function (see formula (17) from section 7.4 of book [8]) and for this reason it is more difficult for calculations. It is more convenient to use the moment method well developed in the transport theory.

\section{SPATIAL MOMENTS METHOD}

The spatial moment of the order $2 k, k=0,1,2, \ldots$ is defined by the integral

$$
m_{2 k}(t)=\int_{-t}^{t} x^{2 k} p_{d}(x, t) d x=2 \int_{0}^{t} x^{2 k} p_{d}(x, t) d x
$$

The moments of odd orders are equal to zero because of the symmetry. It follows from (7) that the moments obey the integral equation

$$
m_{2 k}(t)=m_{2 k}^{(0)}(t)+\sum_{l=0}^{k}\left(\begin{array}{c}
2 k \\
2 l
\end{array}\right) \int_{0}^{t} m_{2 l}^{(0)}(\tau) m_{2(k-l)}(t-\tau) d \tau
$$

Using the Laplace transformation

$$
\tilde{m}_{2 k}(\lambda)=\int_{0}^{\infty} e^{-\lambda t} m_{2 k}(t) d t
$$

we arrive at the following recurrent relation

In the three-dimensional case the density $p_{3}(x, t)$ has a

$$
\tilde{m}_{2 k}(\lambda)= \begin{cases}\lambda^{-1} & , k=0 \\
\frac{(\lambda+1)^{2}}{\lambda^{2}} \tilde{m}_{2}^{(0)}(\lambda) & , k=1 \\
\frac{(\lambda+1)^{2}}{\lambda^{2}} \tilde{m}_{2 k}^{(0)}(\lambda)+\frac{\lambda+1}{\lambda} \sum_{l=1}^{k-1}\left(\begin{array}{c}
2 k \\
2 l
\end{array}\right) m_{2 l}^{(0)}(\lambda) m_{2(k-l)}(\lambda) & , k \geq 2\end{cases}
$$

where (see (A.10))

$$
\tilde{m}_{2 k}^{(0)}(\lambda)=2 \int_{0}^{\infty} d t e^{-(\lambda+1) t} t^{2 k} \cdot \int_{0}^{1} v_{x}^{2 k} W_{d}\left(v_{x}\right) d v_{x}=\frac{\Gamma(2 k+1) \Gamma(d / 2) \Gamma(k+1 / 2)}{\sqrt{\pi} \Gamma(k+d / 2)(\lambda+1)^{2 k+1}}
$$

In particular

$$
\tilde{m}_{2}(\lambda)=\frac{2}{d \lambda^{2}(\lambda+1)}, \quad \tilde{m}_{4}(\lambda)=\frac{(72 \lambda+24) d+48}{(2+d) d^{2} \lambda^{3}(\lambda+1)^{3}}
$$

and so on. On inverting the transforms by means of the residue theorem one obtains the moments $m_{2 k}(t)$. In particular 


$$
\begin{aligned}
m_{0}(t) & =1, \\
m_{2}(t) & =\frac{2}{d}\left[t-1+e^{-t}\right], \\
m_{4}(t) & =\frac{12}{(d+2) d^{2}}\left\{6(4-d)-12 t+(d+2) t^{2}\right. \\
& \left.-\left[6(4-d)-6(d-2) t-2(d-1) t^{2}\right] e^{-t}\right\} .
\end{aligned}
$$

The moments $m_{2}(t)$ and $m_{4}(t)$ conform to $\left\langle r^{2}\right\rangle$ and $\left\langle r^{4}\right\rangle$ obtained in [1] (see formulas (29) and (35) there) because

$$
m_{2 k}(t)=\left\langle r^{2 k}\right\rangle\left\langle V_{x}^{2 k}\right\rangle_{d}
$$

It is convenient to pass from the $x$-distribution density $p_{d}(x, t)$ to the $\xi=x / t$-distribution density

$$
\varphi_{d}(\xi, t) \equiv p_{d}(t \xi, t) t, \quad-1 \leq \xi \leq 1,
$$

with the moments

$$
\int_{-1}^{1} \xi^{2 k} \varphi_{d}(\xi, t) d \xi=\mu_{2 k}(t)
$$

We use the moments for the reconstruction of the density (24) by means of a system of orthogonal on $[-1,1]$ polynomials $f_{m}(\xi), m=0,1,2, \ldots M$ :

$$
\varphi_{d}(\xi, t) \approx \varphi_{d}^{(M)}(\xi, t)=w(\xi, t) \sum_{m=0}^{M} C_{m}(t) f_{m}(\xi) .
$$

Here $C_{m}(t)$ is given by the expression

$$
C_{m}(t)=\int_{-1}^{1} f_{m}(\xi) \varphi_{d}(\xi, t) d \xi
$$

following from the orthogonality of $f_{m}(\xi)$ :

$$
\int_{-1}^{1} w(\xi, t) f_{m}(\xi) f_{n}(\xi) d \xi=\delta_{m n}
$$

Substituting

$$
f_{m}(\xi)=a_{0}+a_{1} \xi+\ldots+a_{m} \xi^{m}
$$

into (26) one can express $C_{m}(t)$ through the moments $\mu_{m}$ and reconstruct $\varphi_{d}(\xi, t)$ by Eq. (25) (notice that $\mu_{m}=0$ for the odd values of $m$ ).

It is known that the closer the weight function $w(\xi)$ to the sought function of $\xi$ the more effective the moment method is, i.e. a lesser number of moments is needed for the reconstruction of the function. To raise the effectiveness we separate the time-axis into the domains: $\left(0, t^{*}\right)$ and $\left(t^{*}, \infty\right)$, where $t^{*}$ is of the order 1 , and use different polynomials in different domains.

At small $t$, the unscattered particles dominate and according to (4)

$$
\begin{aligned}
\varphi_{d}(\xi, t) & \sim W_{d}(\xi) \\
& =\frac{\Gamma(d / 2)}{\sqrt{\pi} \Gamma((d-1) / 2)}\left(1-\xi^{2}\right)^{(d-3) / 2}, \quad d \geq 2 .
\end{aligned}
$$

Therefore, it is reasonable to take here the Gegenbauer (ultraspherical) polynomials

$$
G_{m}^{(\alpha)}(\xi)=\frac{1}{\Gamma(\alpha)} \sum_{k=0}^{[m / 2]}(-1)^{k} \frac{\Gamma(\alpha+m-k)}{k !(m-2 k) !}(2 \xi)^{m-2 k}
$$

that have the weight function

$$
w(\xi)=\left(1-\xi^{2}\right)^{\alpha-1 / 2} .
$$

The symbol $[\mathrm{m} / 2]$ denotes an integer part of $m / 2$. As a result we get for $t<t^{*}$

$$
\varphi_{d}^{(M)}(\xi, t)=\left(1-\xi^{2}\right)^{\alpha-1 / 2} \sum_{m=0}^{M} C_{m}(t) G_{m}^{(\alpha)}(\xi),
$$

where

$$
\begin{aligned}
C_{m}(t) & =\frac{m !(m+\alpha)}{\pi 2^{1-2 \alpha}} \frac{\Gamma(\alpha)}{\Gamma(m+2 \alpha)} \\
& \times \sum_{k=0}^{[m / 2]}(-1)^{k} \frac{\Gamma(\alpha+m-k)}{k !(m-2 k) !} 2^{m-2 k} \mu_{m-2 k}(t)
\end{aligned}
$$

and $\alpha=d / 2-1$.

At large $t$ the diffusion regime arises

$$
\varphi_{d}(\xi, t) \sim \frac{1}{\sqrt{2 \pi \mu_{2}}} e^{-\xi^{2} /\left(2 \mu_{2}\right)} .
$$

Hence, the Hermitian polynomials

$$
H_{m}(\xi)=m ! \sum_{k=0}^{[m / 2]}(-1)^{k} \frac{\sigma^{-2(m-k)}}{k ! 2^{k}(m-2 k) !} \xi^{m-2 k}
$$

are more appropriate here since their weight functions are just (28). In this case

$$
\varphi_{d}^{(M)}(\xi, t)=\frac{1}{\sqrt{2 \pi} \sigma} e^{-\frac{\xi^{2}}{2 \sigma^{2}}} \sum_{m=0}^{M} C_{m}(t) H_{m}(\xi)
$$


with

$$
C_{m}(t)=\sum_{k=0}^{[m / 2]}(-1)^{k} \frac{\sigma^{2 k}}{2^{k} k !(m-2 k) !} \mu_{m-2 k}(t)
$$

and

$$
\sigma^{2}=\mu_{2}(t)
$$

The results of our calculations of $\varphi_{d}(\xi, t), d=3,4,5$, by formula (27) for $t=3$ and by formula (29) for $t=10,30$ and 100 are presented in Figs. $1-4$. The results of the telegraph approximation and of the diffusion approximations are shown there too.

\section{CONCLUSION}

What did we expect to see from the comparison? Remember that

i) the diffusion equation is derived from the telegraph equation omitting the term with the second timederivative (compare (15) with (18));

ii) like the kinetic solution the telegraph solution has a diffusion front beyond which the walking particle cannot appear, whereas the diffusion solution stretches up to infinity at any time $t$;

iii) the second moment of the telegraph solution exactly coincides with the kinetic solution at any time, whereas the second diffusion moment differs from the exact one and coincides with it only in asymptotic $t \rightarrow \infty$ (see (23), (B.4) and (17));

$i v)$ in the one-dimensional case $(d=1)$ the telegraph equation gives the exact solution of the kinetic problem whereas the diffusion equation stays approximate.

Thus it was natural to expect for $d \geq 2$ the telegraph equation to give the results that are more exact, i.e. closer to the solution of the kinetic equation than the diffusion result.

However, the numerical results presented in Figs. 1-4 lead to quite opposite conclusion for $d>2$. The solution of the telegraph equation turns out to be farther from the exact result than the solution of the diffusion equation. The cause lies in the structure of the telegraph equation according to which the front of the distribution is situated in the point $1 / \sqrt{d}$ corresponding to the meansquare-root velocity projection on the $x$-axis, whereas the exact position of the front is $t$. Thus the higher the dimension $d$ the more the two positions differ.

To obtain an approximate solution closer to the exact solution than diffusion approximation one have to use $P_{N}$-approximations of higher orders $(N>1)$ which will be investigated in our next works.

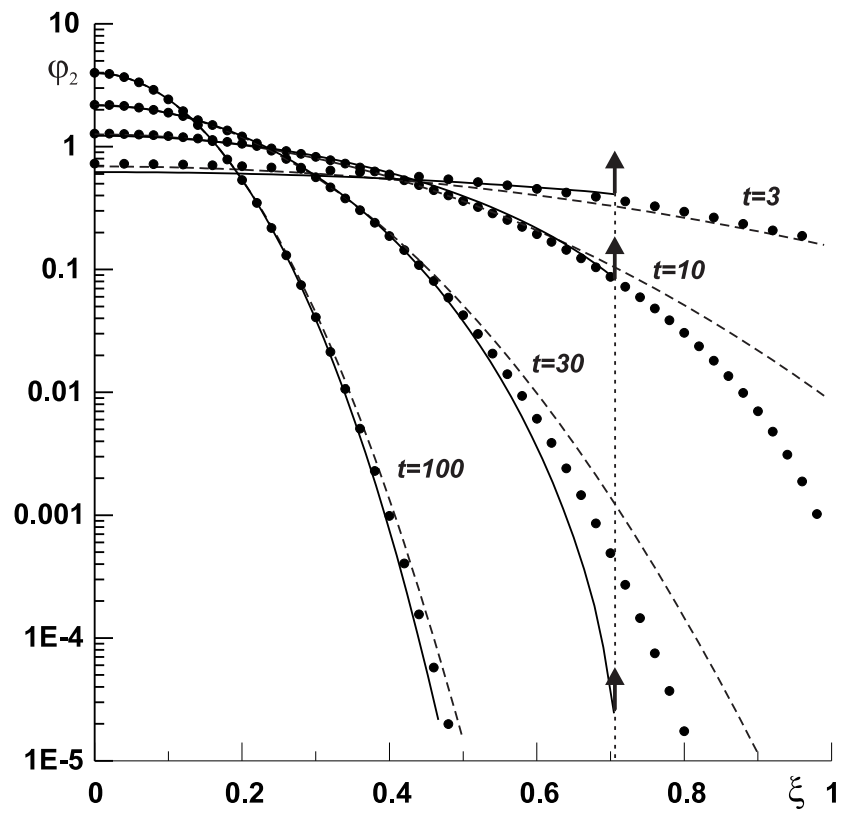

Fig. 1. Distribution density $\varphi_{d}(\xi, t)=p_{d}(t \xi, t) t$ for different $t$. Filled circles present the solution of the kinetic equation (7) obtained by (22) for $d=2$; the dashed lines are the solution of the diffusion equation (18) and the solid lines show the solution of the telegraph equation. The vertical arrows indicate the position of the singularity in (16) coinciding with the front positions.

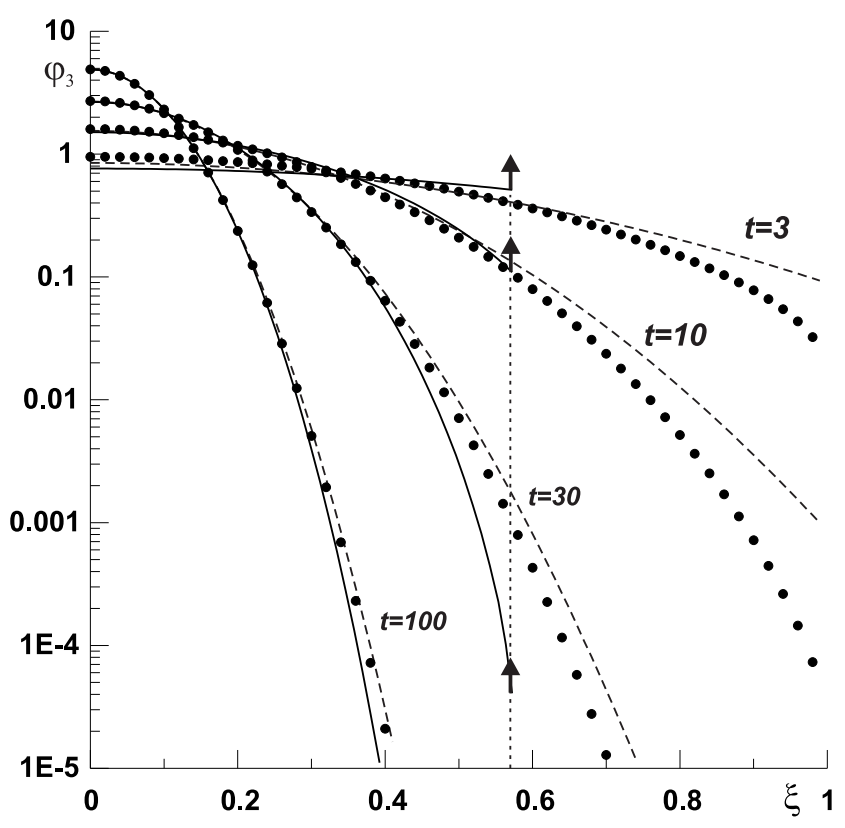

Fig. 2. Distribution density $\varphi_{d}(\xi, t)=p_{d}(t \xi, t) t$ for different $t$. Filled circles present the solution of kinetic equation (7) reconstructed from the moments $\mu_{0}, \mu_{2}, \ldots, \mu_{10}$, for $d=3$; the dashed lines are the solution of the diffusion equation (18) and the solid lines show the solution of the telegraph equation. The vertical arrows indicate the position of the singularity in (16) coinciding with the front positions. 


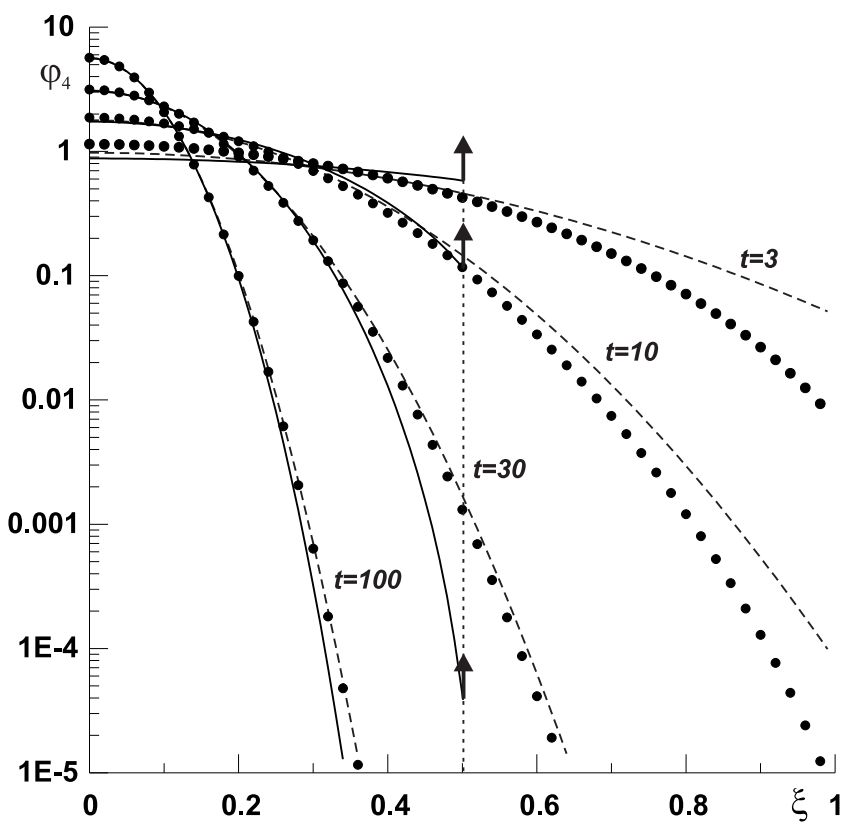

Fig. 3. Same as Fig. 2 for $d=4$.

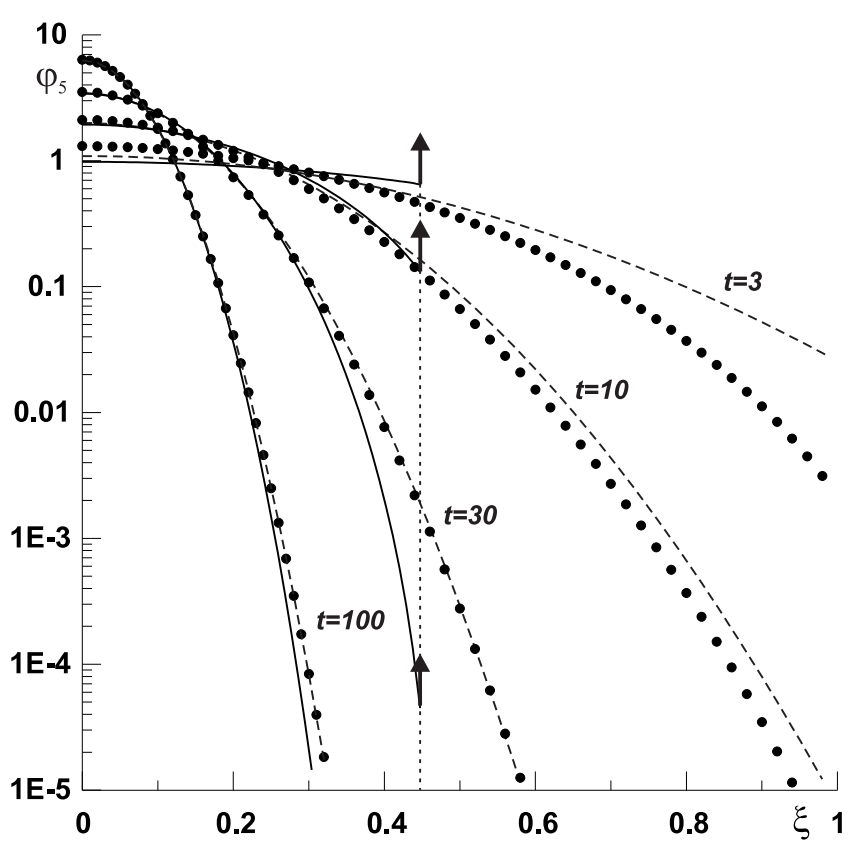

Fig. 4. Same as Fig. 2 for $d=5$.

\section{ACKNOWLEDGEMENTS}

We are grateful to G.Gusarov for preparing the manuscript.

\section{APPENDIX A. CALCULATION OF $W_{d}\left(v_{x}\right)$ $\operatorname{AND}\left\langle V_{x}^{2 k}\right\rangle_{d}$}

The two known integrals

$$
\int_{\mathrm{R}^{d}} f\left(\mathbf{x}^{2}\right) d \mathbf{x}=\frac{2 \pi^{d / 2}}{\Gamma(d / 2)} \int_{0}^{\infty} t^{d-1} f\left(t^{2}\right) d t
$$

and

$$
\begin{aligned}
& \int_{\mathrm{R}^{d}} f\left(x^{2}\right) h(\mathbf{a x}) d \mathbf{x}=\frac{2 \pi^{(d-1) / 2}}{\Gamma((d-1) / 2)} \\
& \times \int_{0}^{\infty} d t t^{d-1} f\left(t^{2}\right) \int_{-1}^{1} h(|\mathbf{a}| t \mu)\left(1-\mu^{2}\right)^{\frac{d-3}{2}} d \mu
\end{aligned}
$$

will be used below.

The distribution density $p_{d}(\mathbf{x})$ of the unit isotropic $d-$ dimensional vector is written as follows:

$$
p_{d}(\mathbf{x})=C_{d} \delta\left(x^{2}-1\right)
$$

where $\delta(z)$ is the one-dimensional Dirac's function and $C_{d}$ is a constant found from normalization

$$
\int_{\mathrm{R}^{d}} p_{d}(\mathbf{x}) d \mathbf{x}=1
$$

On substituting (A.3) in (A.4) and using (A.1) we obtain

$$
C_{d}=\frac{\Gamma(d / 2)}{\pi^{d / 2}}
$$

It is evident that for $d=1$

$$
W_{1}\left(v_{x}\right)=(1 / 2)\left[\delta\left(v_{x}-1\right)+\delta\left(v_{x}+1\right)\right] .
$$

For $d>1$ we calculate the distribution function

$$
F_{d}\left(v_{x}\right)=\int_{-1}^{v_{x}} W_{d}\left(v_{x}^{\prime}\right) d v_{x}^{\prime}
$$

According to its definition

$$
F_{d}\left(v_{x}\right)=\int_{\mathrm{R}^{d}} p_{d}(\mathbf{x}) H\left(v_{x}-\mathbf{e x}\right) d \mathbf{x}
$$

where $\mathbf{e}$ is the unit vector directed along one of the axes. Setting (A.3) with (A.5) into (A.7) and using (A.2) we have got 


$$
\begin{aligned}
& F_{d}\left(v_{x}\right)=\frac{\Gamma(d / 2)}{\sqrt{\pi} \Gamma((d-1) / 2)} \int_{0}^{\infty} d \tau \tau^{(d-1) / 2} \\
& \times \int_{-1}^{1} \delta(\tau-1) H\left(v_{x}-\sqrt{\tau} \mu\right)\left(1-\mu^{2}\right)^{(d-3) / 2} d v_{x} \\
& =\frac{\Gamma(d / 2)}{\sqrt{\pi} \Gamma((d-1) / 2)} \int_{-1}^{1} H\left(v_{x}-\mu\right)\left(1-\mu^{2}\right)^{(d-3) / 2} d v_{x} .
\end{aligned}
$$

Differentiating (A.8) with respect to $v_{x}$ and taking into account (A.6) and the relation

$$
d H(t) / d t=\delta(t)
$$

we get

$$
W_{d}\left(v_{x}\right)=\frac{\Gamma(d / 2)}{\sqrt{\pi} \Gamma((d-1) / 2)}\left(1-v_{x}^{2}\right)^{(d-3) / 2}
$$

and

$$
\begin{aligned}
\left\langle V_{x}^{2 k}\right\rangle_{d} & =\frac{\Gamma(d / 2)}{\sqrt{\pi} \Gamma((d-1) / 2)} 2 \int_{0}^{1} v_{x}^{2 k}\left(1-v_{x}^{2}\right)^{(d-3) / 2} d v_{x} \\
& =\frac{\Gamma(d / 2) \Gamma(k+1 / 2)}{\sqrt{\pi} \Gamma(k+d / 2)}
\end{aligned}
$$

\section{APPENDIX B. SOLUTION OF THE}

\section{TELEGRAPH EQUATION AND ITS MOMENTS}

We denoted above by $g^{(\nu)}(x, t)$ the solution of the telegraph equation (1) satisfying the initial conditions

$$
g^{(\nu)}(x, 0)=\delta(x), \quad\left[\partial g^{(\nu)}(x, t) / \partial t\right]_{t=0}=0
$$

and the conditions at infinity

$$
\begin{gathered}
\qquad \lim _{|x| \rightarrow \infty} g^{(\nu)}(x, t)=0, \\
\lim _{|x| \rightarrow \infty}\left[\partial g^{(\nu)}(x, t) / \partial x\right]=0 . \\
\text { The solution differs from zero only on the it has the form } \\
{[-\nu t, \nu t] \text { where }} \\
g^{(\nu)}(x, t)=\frac{1}{2}[\delta(x-\nu t)+\delta(x+\nu t)] e^{-t / 2} \\
+\frac{1}{4 \nu}\left[I_{0}\left(\sqrt{\left(t^{2}-x^{2} / \nu^{2}\right) / 4}\right)\right.
\end{gathered}
$$

The solution differs from zero only on the segment

$$
\left.+t I_{1}\left(\sqrt{\left(t^{2}-x^{2} / \nu^{2}\right) / 4}\right) / \sqrt{t^{2}-x^{2} / \nu^{2}}\right] e^{-t / 2} .
$$

Here $I_{0}$ and $I_{1}$ stand for the Bessel functions of imaginary argument:

$$
I_{\mu}(z)=(z / 2)^{\mu} \sum_{k=0}^{\infty} \frac{(z / 2)^{2 k}}{k ! \Gamma(\mu+k+1)}
$$

Even moments

$$
m_{2 k}^{(\nu)}(t)=2 \int_{-\nu t}^{\nu t} x^{2 k} g^{(\nu)}(x, t) d x
$$

can be calculated by means of (B.2) and of the integral

$$
\int_{0}^{t}\left(t^{2}-z^{2}\right)^{k} z^{2 n} d z=\frac{\Gamma(n+1 / 2) \Gamma(k+1)}{2 \Gamma(n+k+3 / 2)} t^{1+2(k+n)} .
$$

They have the form

$$
\begin{aligned}
m_{2 k}^{(\nu)}(t) & =(2 \nu)^{2 k} t^{k+1 / 2} e^{-t / 2} \Gamma(k+1 / 2)\left[I_{k+1 / 2}(t / 2)\right. \\
& \left.+I_{k-1 / 2}(t / 2)\right] / 2
\end{aligned}
$$

In particular

$$
\begin{gathered}
m_{0}^{(\nu)}(t)=1, \\
m_{2}^{(\nu)}(t)=2 \nu^{2}\left[t-1+e^{-t}\right] \sim 2 \nu^{2} t, \quad t \rightarrow \infty \\
m_{4}^{(\nu)}(t)=12 \nu^{4}\left[6-4 t+t^{2}-2(3+t) e^{-t}\right] .
\end{gathered}
$$

At large $t$

$$
g^{(\nu)}(x, t) \sim \frac{1}{2 \nu \sqrt{\pi t}} e^{-x^{2} /\left(4 \nu^{2} t\right)}
$$

and the moments (B.3) take the asymptotical form

$$
m_{2 k}^{(\nu)}(t) \sim \frac{(2 \nu)^{2 k}}{\sqrt{\pi}} \Gamma(k+1 / 2) t^{k}
$$

In particular

$$
m_{2}^{(\nu)}(t) \sim 2 \nu^{2} t
$$

and

$$
m_{4}^{(\nu)}(t) \sim 12 \nu^{4} t^{2}
$$

The results (B.5)-(B.8) relate to the diffusive approximation based on the diffusion equation (2). 
[1] R. Garcia-Pelayo, Physica A 216, 299 (1995).

[2] A. G. Webster, Partial Differential Equations of Mathematical Physics (Hafner, New York, 1950).

[3] S. Goldstein, Quar. J. Mech. Appl. Math. IV Pt. 2, 129 (1950).

[4] A. S. Monin, Izv. Akad. Nauk SSSR, Ser. Geofiz. No. 3, 234 (1955) (In Russian).

[5] A. M. Weinberg, L. C. Noderer, Theory of Neutron Chain Reactions (AECD-3471, 1951).

[6] A. M. Weinberg, E. P. Wigner, The Physical Theory of Neutron Chain Reactors (Univ. of Chicago Press, Chicago, Illinois, 1958).

[7] P. B. Daitch, D. B. Ebeoglu, Nucl. Sci. Eng. 17, 212
(1963).

[8] K. M. Case, P. F. Zweifel, Linear Transport Theory (Addinson-Wesley, Reading, MA, 1967).

[9] S. L. Sobolev, Usp. Fiz. Nauk 167, 1095 (1997).

[10] S. Godoy, L. S. Garcia-Colin, Physica A 258, 414 (1998).

[11] Handbook of Mathematical Functions, edited by M. Abramowitz, I. Stegun (NBS Appl. Math. Ser. 55, 1964).

[12] W. Feller, An Introduction to Probability Theory and its Applications, Vol. II (Wiley, New York, 1966).

[13] J. Masoliver, J. M. Porrá, G. H. Weiss, Physica A 193, 469 (1993).

\title{
ТЕЛЕГРАФНЕ РІВНЯННЯ В ЗАДАЧІ ПРО ВИПАДКОВІ БЛУКАННЯ
}

\author{
В. В. Учайкін, В. В. Саєнко \\ Ульяновсъкий дерюсавний університет, Інститут теоретичной фізики, \\ Ульяновсък, 432700, Росія \\ e-mail: uchaikin@sv.uven.ru
}

\begin{abstract}
Розглянуто ізотропне випадкове блукання частинки зі сталою швидкістю в $d$-вимірному просторі. Шей процес описаний кінетичним рівнянням, що розв'язується в термінах квадратур чи спещіяльних функцій лише при $d=1$ та 2. Для $d>2$ використовуються , пві скорочені форми рівняння - телеграфне рівняння та рівняння дифузіі. Друге з них звичайно вважають гіршим наближенням, ніж перше. Проведені числові дослідження насправді вказують на протилежну ситуацію: для $d \geq 2$ простий результат дифузійного рівняння виявляється ближчим до точного результату, ніж складніший результат телеграфного рівняння.

Отримані результати застосовані до задачі поверхневого та об'ємного переносу. Вони придатні для опису хаотичної динаміки системи в термінах випадкових блуканњ у фазовому просторі.
\end{abstract}

